\title{
A Novel Based Approach For Pose Estimation And Normalization Using Multi-Class Svm And Affine Transformation For Face Recognition
}

\author{
${ }^{1}$ shilpa R, ${ }^{2}$ shreekumar T \\ ${ }^{I}$ M.Tech, CNE, Mangalore Institute of Technology and Engineering, Moodbidri, Karnataka \\ ${ }^{2}$ Associate Professor, CSE, Mangalore Institute of Technology and Engineering, Moodbidri, Karnataka
}

\begin{abstract}
The goal of the paper is to estimate the pose, normalize the pose, and remove the illumination to recognize the face. The training section includes the techniques Affine Transformation and DCT. During the testing section the estimation of pose is calculated and later normalized frames of the pose variant frames and illumination variant frames of the testing person are obtained by using Affine Transformation and DCT methods respectively. Once the normalization and illumination removal methods are done, face recognition is carried out. The features are extracted using the principle component analysis; later face recognition is carried out by matching the obtained features with the database features. Once the normalization and illumination removal methods are done, face recognition is carried out. The features are extracted using the principle component analysis; later face recognition is carried out by matching the obtained features with the database features.
\end{abstract}

Keywords: Support Vector Machine, Affine Transformation, Discrete Cosine Transformation, Principal Component Analysis.

\section{Introduction}

In the recent years, the research based on face recognition has been significantly increased. Research works have focused on factors like illumination, pose, identity, facial expression, hair style, aging, make-up, scale etc $[1,2]$. Out of these factors the main problems are illumination and pose. Disadvantage of illumination is computational cost. Storing large number of views of each image is a difficult task. Varying illumination and pose normalization is the challenging problem. Variations between the images of the same face due to illumination are almost always larger than the image variations due to change in face identity [3]. So, it is difficult for the computer to do the face recognition when the input images and gallery images are different. And in recent years SVM classifier is used often because it gives superior recognition rate compared to other classifiers. SVM is mainly designed for binary decision problems. Multi-class SVM is used to solve the pose estimation problem.Decomposing the multi-class problems into several two-class problems is the popular method of using multi-class SVM [7].

In the proposed system the modification is done using two stages, training section and testing section. In the training section, the database is trained using Affine Transformation and DCT. In the testing section the image of the person to be recognized is given as input. Pose estimation is calculated by using Multi Class SVM algorithm [7]. The input may or may not have the pose and illumination variation. However the normalized image of the person is obtained using Affine Transformation [4][11][13] and DCT [5][10][13] techniques subsequently used for the dimension reduction and feature vector. The recognition is done by PCA algorithm [6] [14] to get the good accuracy. The proposed system is given in section 2. The proposed approaches are presented in Section 3.Section 4 includes experimental result and conclusion is given in section 5. Finally, the acknowledgement is given in section 6 .

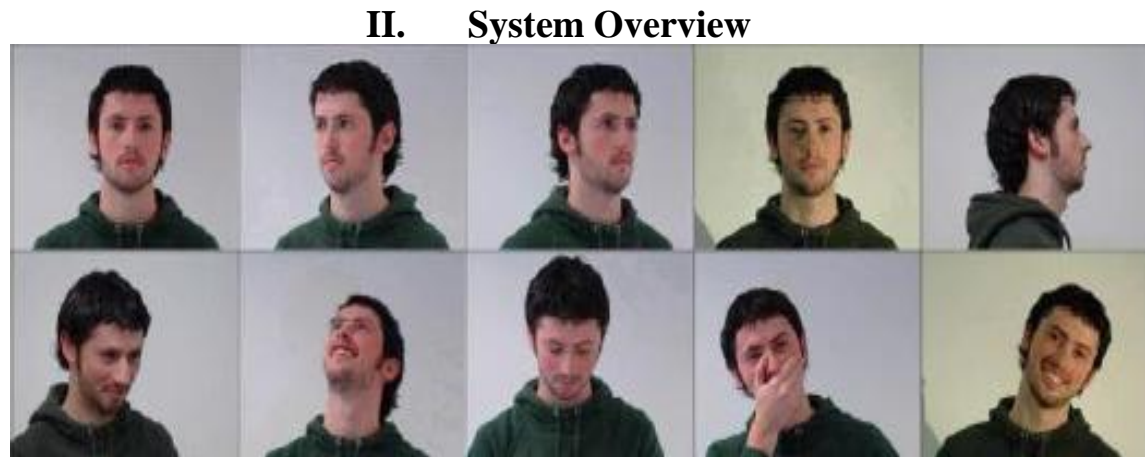

Fig. 1: The samples from the database 


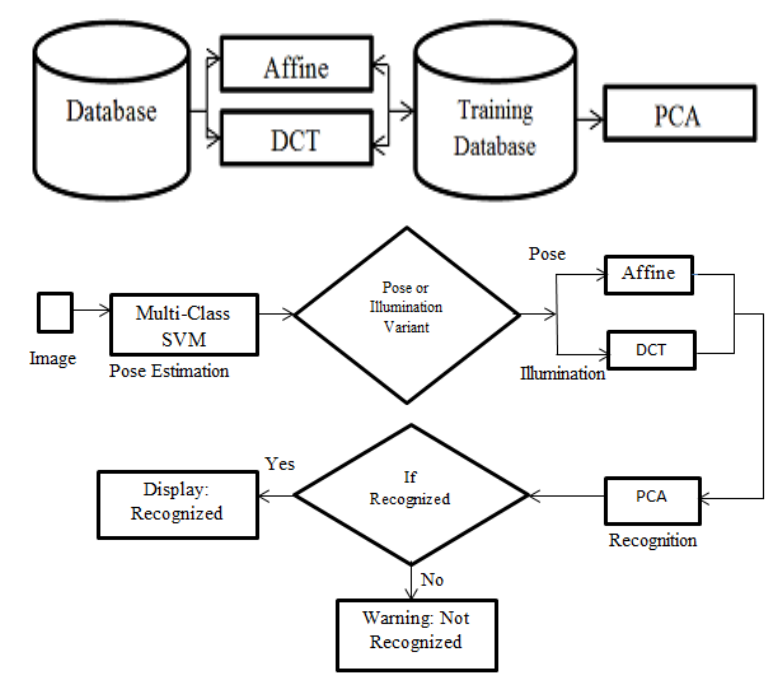

Fig. 2: Process of proposed system

In the proposed system face images of a single person with different pose are taken. The face samples for normalization and illumination are shown in Fig. 1. The goal is to normalize the pose using Affine Transformation and remove the illumination using DCT are done first. The system overview is shown in Fig. 2. At first the pose estimation is carried out by Multi-Class SVM method. Then normalization is done using Affine transformation to a non-frontal image. And illumination removal method is done using DCT algorithm [12]. Once the normalization technique is applied, all the images of different class are normalized to the frontal view. All classes of images are normalized to the same image size and the same distance between two eyes. The precise locations of irises can be got by the region growing search method [8]. Then illumination effect is removed. Once these two processes are completed an image is given for recognition. Face recognition process is done using PCA algorithm. Success result is then shown. In order to get the good accuracy and decrease the computation cost these algorithms are used.

\subsection{Support Vector Machine for pose estimation}

\section{Algorithms Used}

The support vector machine is originally a binary classification method [15] [16]. There are varieties of techniques used for the classification in SVM. They are One-against-all (OvA) and One-against-one (OvO). Consider there are many images which are arranged in a binary tree structure [17]. A SVM in each node (i.e. images) of the tree is trained using two of the classes. The algorithm then employs to measure the similarity between the remaining samples and two classes used for training. All samples in the node are assigned to the two sub nodes derived from the previously selected classes by similarity. This step repeats at every node until each node contains only samples from one class. The main problem is the training time which decreases the performance. An example of SVM that solves a 7 - class pattern recognition problem utilizing a binary tree, in which each node makes binary decision using a SVM, is shown on Fig. 3. The hierarchy of binary decision subtasks should be carefully designed before the training of each SVM classifier.

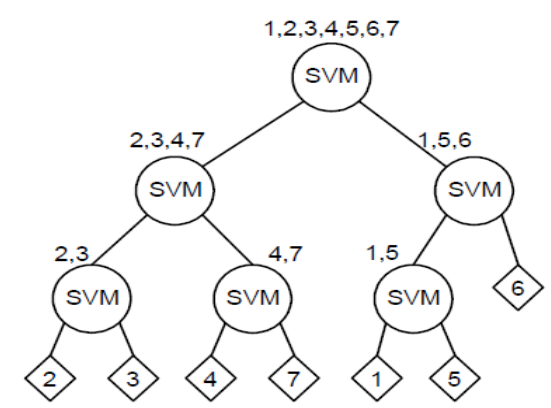

Fig. 3: Illustration of SVM binary tree.

The SVM method that proposed is based on recursively dividing the classes in two disjoint groups in every node of the decision tree and training a SVM that will decide in which of the groups the incoming unknown sample should be assigned. The groups are determined by a clustering algorithm according to their 
class membership. For the training set $\left(\mathrm{x}_{1}, \mathrm{y}_{1}\right) \ldots\left(\mathrm{x}_{\mathrm{n}}, \mathrm{y}_{\mathrm{n}}\right)$ with labels $\mathrm{y}_{\mathrm{i}}$ in $[1 \ldots \mathrm{k}]$, it finds the solution of the following optimization problem during training.

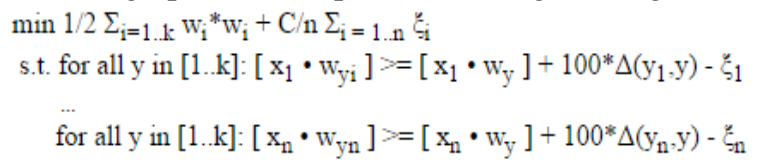

\subsection{Affine Transformation for pose normalization}

A function between affine spaces which preserves points, straight lines and planes is called as affine transformation. It is a combination of translation and linear map. Affine Transformation in two dimensional includes translation, scaling, rotation, shear mapping. The method can be describes as follows, face region is divided into three rectangles. Comparison between original image and specific pose are calculated. The normalized face image in some pose can be geometrically warped into the frontal pose. Having the virtual frontal pose view, then the DCT algorithm can be used to remove the illumination effect of an image. The formula description is given below;

1) Two rectangle's 8 vertices are given $\left(R_{l}, R_{o}\right)$ under left pose and original front pose.

2) For the 4 vertices $\left(u_{i}, v_{i}\right) i=1,2,3,4$ in $R_{o}$, after the transformation, corresponding 4 vertices are obtained ( $u_{i}^{\prime}$, $\left.v_{i}^{\prime}\right) i=1,2,3,4$.

3) Let's use $\mathrm{Xo}, \mathrm{A}, \mathrm{X}_{1}$ denote the three matrices and together it is written as;

Xo . $A=X_{1}$

So the parameter can be calculated by;

$\mathrm{A}=\mathrm{Xo}^{-1} \cdot \mathrm{X}_{\mathrm{l}}$

4) Using there parameters, virtual frontal view is obtained. For each point $(u, v) u=i n$ the frontal view, first rectangle is determined, then two parameters are decided and mapping of an image is done. Define ( $\left.u^{\prime}, v^{\prime}\right)$ as the transformed value $(\mathrm{u}, \mathrm{v})$ and expressed as,

$\left\{\mathrm{u}^{\prime}=\mathrm{ao}+\mathrm{a} 1 \cdot \mathrm{u}\right.$

$\left\{v^{\prime}=v\right.$

Let $\left\{\begin{array}{c}u 1=\operatorname{int}\left(u^{\prime}\right) \\ v 1=v^{\prime}\end{array}\right.$ and $\mathrm{du}=\mathrm{u}^{\prime}-\operatorname{int}\left(\mathrm{u}^{\prime}\right)$

So, $\mathrm{f}(\mathrm{u}, \mathrm{v})$ can be written as,

$f(u, v)=(1-d u) \cdot f^{\prime}(u 1, v 1)+d u . f^{\prime}(u 1+1 . v 1)$, where $f(u, v)$ is given to frontal view and $f^{\prime}(u, v)$ is a value given to non-frontal view.

\subsection{Discrete Cosine Transformation for illumination removal}

DCT was categorized into four slightly different transformations named DCT-I, DCT-II, DCT-III, and DCT-IV [9]. The DCT is generally used in image processing and signal, mainly for lossy compression, because it has a steady energy compaction property. DCT will take the input, it does partition based on the co-efficient. After obtaining the coefficients, low frequency and high-frequency are separately modified to remove the illumination effect. After the removal of illumination effect details of the images are highlighted. The modified coefficients are used in order to reconstruct the new image via inverse discrete cosine transform. The reconstructed image is used for recognition process.

The 2D DCT of $\mathrm{x}$ matrixis defined as follows;

$$
y(k)=w(k) \sum_{n=1}^{N} x(n) \cos \left(\frac{\pi(2 n-1)(k-1)}{2 N}\right) \quad k=1,2, \ldots N
$$

Where

$$
w(k)= \begin{cases}\frac{1}{\sqrt{N}} & k=1 \\ \sqrt{\frac{2}{N}} & 2 \leq k \leq N\end{cases}
$$

$\mathrm{N}$ is the length if the matrix $\mathrm{x}$ and $\mathrm{x}, \mathrm{y}$ are the same size.

\subsection{Principal Component Analysis for face recognition}

Principal component analysis (PCA) is a statistical conversion process using orthogonal transform. It is possible to display a set of related variables. It is non-correlated linearly to set the value of the variable so-called 
principal component. Uncorrelated orthogonal basis set are obtained as resulting vector. The PCA algorithm extracts the face features, and reduces the dimensionality of input space. Variations between the images are seen due to the variations of pose and illumination. So the recognition rate obtained is very high which provides good accuracy. Fig. 4 shows the face recognition system.

"Face Recognition" generally involves two stages:

1. Face Detection: where a photo is searched to find any face, then image processing cleans up the facial image for easier recognition.

2. Face recognition: where that detected and processed face is compared to a database of known faces, to decide who that person.

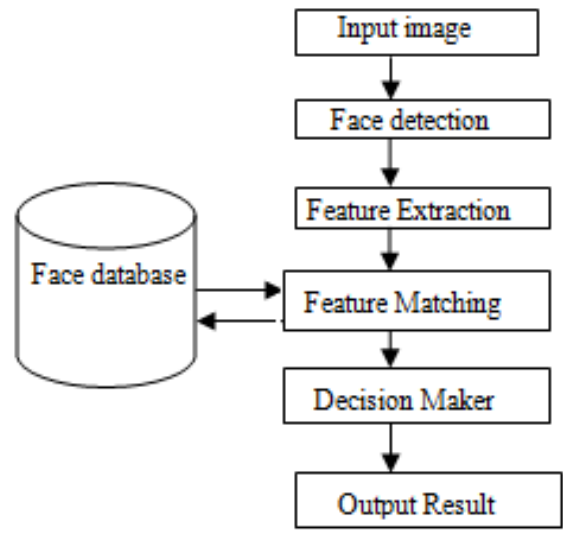

Fig. 4: Face recognition system

PCA has been extensively employed for face recognition algorithms. It not only reduces the dimensionality of the image, but also retains some of the variations in the image data. Face image onto a feature space that spans the significant variations. The significant features are known as eigenvectors (principle component) of the set of faces they do not necessarily correspond to the features such as eyes, ears and noses. So to recognize a particular face it is necessary only to compare these weights to those individuals.

\section{Experimental Analysis}

The proposed Video face recognition system is implemented in MATALB platform and it is evaluated using the dataset of FERET Face Database. All face images are normalized according to the different criterions associated with different pose classes. All the frames in the database are masked. Fig. 5 represents the sample frames set for normalization process and the Fig. 6 represents the sample frames set for lamination variant process.

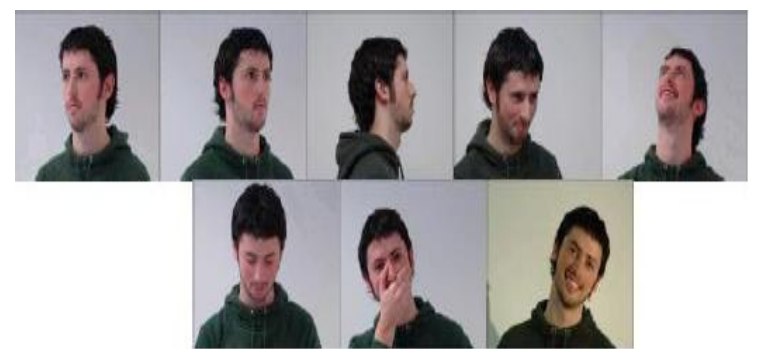

Fig. 5: Sample frameset for normalization process

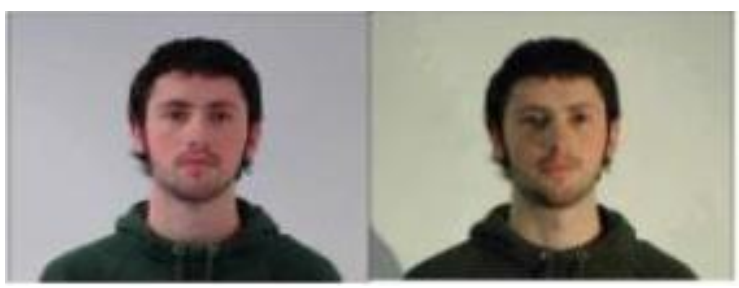

Fig. 6: Sample frameset for illumination variant process

In the normalization process the normalized frames are obtained by Affine Transformation method and in the illumination invariant process the removal of illumination effect images are obtained by DCT. Fig. 7 
represents the normalized image after normalization process and Fig. 8 represents the illumination removal image after illumination invariant process subsequently, obtained normalized images are subjected to face recognition using PCA.

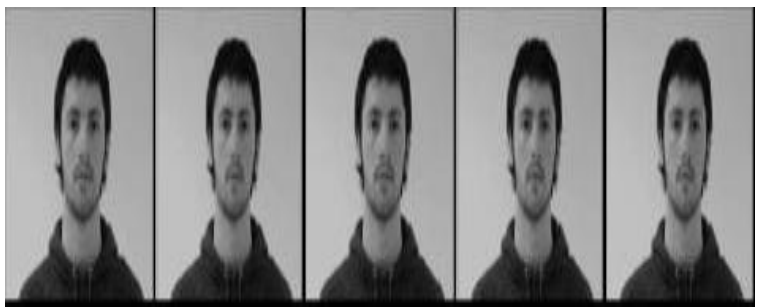

Fig. 6: Normalized face images after Affine Transformation

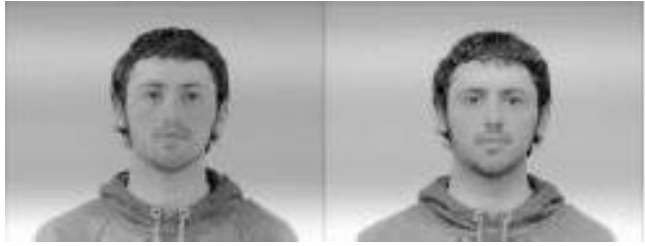

Fig. 6: Illumination effect removal after DCT

\section{Conclusion}

The pose normalization algorithm based on the statistical transformation is presented in this paper. The proposed algorithm can solve the pose-invariant face recognition problem, especially when face rotation angle is less than 30 degree. Experiments show that the method has a good performance for normalize the non-frontal face images, and can dramatically increase the recognition rate with a very low time complexity. The results of the pose estimation show that the speed of training and testing are improved, while keeping comparable or offering better recognition rates than the other SVM multi-class methods. The experiments showed that this method becomes more favourable as the number of classes in the recognition problem increases.The deal with the illumination problem to make face recognition robust to illumination variations is given in this paper. This method is based on the modification of discrete cosine transform coefficients. The experimental results show that $100 \%$ face recognition rate was achieved on FERET Datasets. Then PCA method is used for face recognition process. Facial features are extracted by the PCA method, which reduces the dimensionality of the original face images while preserving some discriminating features within the training images. After performing the PCA, structural information is acquired corresponding to each person from lower dimensional training images. The experimental results obtained on both the face databases are found to be quite promising and better than some of the PCA-based methods reported earlier.

\section{Acknowledgments}

Dedicating the paper work to my esteemed guide;Associate Professor, Mr Shreekumar T, whose interest and guidance helped in the completion of the work successfully and also extending the gratitude to Prof. Dr Nagesh H R (H.O.D Computer Science and Engineering Department) who has facilitated exploring the subject with more enthusiasm.

\section{References}

[1]. Wu-Jun Li, Chong-Jun Wang, Dian-Xiang Xu and Shi-Fu Chen, "Illumination Invariant Face Recognition Based on Neural Network Ensemble", In Proceedings of 16th IEEE International Conference on Tools with Artificial Intelligence, pp. 486-490, November 2004

[2]. Majumdar and Ward, "Pseudo-Fisher face Method for Single Image Per Person Face Recognition", In Proceedings of IEEEInternational Conference on Acoustics, Speech and Signal Processing, Las Vegas, NV, pp. 989-992, 2008.

[3]. D. Beymer and T. Poggio. Face Recognition From One

[4]. Example View. ICCV, Boston, MA, pp. 500-507, 1995

[5]. Xiujuan Chai1, Shiguang Shan, Wen Gao, "Pose normalization for robust face recognition using Statistical Affine Transformation", In proceeding of ICICS- PCM 2003, 15-18 Dec 2003, Singapore.

[6]. Shan Du, Mohamed Shehata, WalBadawy, "A novel algorithm for illumination invariant DCT- based face recognition", IN proceeding with 25th IEEE Canadian Conference on Electrical and Computer Engineering (CCECE), 2012.

[7]. S. Thakur, J. K. Sing, D. K. Basu, M. Nasipuri, M. Kundu, "Face recognition using principal component analysis and RBF- Neural network". IJSSST, Vol. 10, No. 5.

[8]. GjorgjiMadzarov, DejanGjorgjevikj and Ivan Chorbev, "A Multi-class SVM Classifier Utilizing Binary Decision Tree”, Informatica 33 (2009) 233-241 233.

[9]. B. Cao, S. Shan,W. Gao. Localizing the iris center by region growing search. Proceeding of the ICME2002. 
[10]. Z. Wang, "Fast algorithms for the discrete W transform and for the discrete Fourier transform," IEEE Trans. On Acoustic, Speech, and Signal Processing, vol. 32, pp. 803-816, 1984.

[11]. Shreekumar T and Nagaratna P. Hegde, "AN APPROACH TO POSE AND ILLUMINATION INVARIANT FACE RECOGNITION IN VIDEO”, International Journal of Image Processing and Applications, 2(1), 2011, pp. 93-100.

[12]. Shreekumar T, Karunakara K, "Particle Swam Optimization Based Identification of Face Images from Video", International Journal of Advanced Computational Engineering and Networking, ISSN: 2320-2106, Volume-4, Issue-2, Feb.-2016.

[13]. Shreekumar T, Dr. NagaratnaHegde, "A New Method For Face Recognition From Video Sequence Using DCT and LLR", Internal journal of System, algorithms \& application, Volume 2, Issue ICRAET12, May 2012, ISSN Online: 2277-2677.

[14]. Mahesh Prasanna K, NagaratnaHegde, "A Fast Recognition Method for Pose and Illumination Variant Faces on Video Sequences", IOSR Journal of Computer Engineering (IOSR-JCE) e-ISSN: 2278-0661, p- ISSN: 2278-8727Volume 10, Issue 1 (Mar. - Apr. 2013), PP 08-18 www.iosrjournals.org.

[15]. Amith G K, Shreekumar T, "A Novel Approach for Blurred and Noisy Face Image Recognition", International Journal of Science and Research (IJSR) ISSN (Online): 2319-7064.

[16]. V. Vapnik. The Nature of Statistical Learning Theory, 2nd Ed. Springer, New York, 1999.

[17]. C. J. C. Burges. A tutorial on support vector machine for pattern recognition. Data Min. Knowl. Disc. 2 (1998) 121.

[18]. B. Fei, J. Liu. Binary Tree of SVM: A New Fast Multiclass Training and Classification Algorithm. IEEE Transaction on neural networks, Vol. 17, No. 3, May 2006. 